\title{
Feasibility of a novel two-piece nasogastric feeding tube for patients with dysphagia
}

\author{
Sen-Yung $\underline{\mathrm{Liu}}^{1,3}$, MD, Chao-Pin $\underline{\text { Yang}}^{1}$, MD, Ta-Sen $\underline{\text { Wei }}^{1}$, MD, Yen-Chun $\underline{\text { Chen }}{ }^{1}$, MD, Chih-Hao $\underline{\text { Liang }}^{1}$, MD,
} Ching-Hsuan $\underline{W u}^{1}$, MD, Chih-Lin $\underline{C h e n}^{2}$, MD, Tsung-Ju $\underline{W u^{1}}$, MD

INTRODUCTION The exposed section of a traditional nasogastric (NG) tube can interfere with patients' social activities and thereby result in distress. This study was conducted to evaluate the feasibility and safety of a novel two-piece NG tube for patients with dysphagia.

METHODS Ten patients with dysphagia were recruited between November 2011 and May 2012. Patients who were unconscious or in critical condition, had a traditional NG tube $<50 \mathrm{~cm}$ or $>60 \mathrm{~cm}$ in fixed length, or were unable to follow instructions or sign consent forms were excluded. The two-piece NG tube, which was placed in the patients for one week, comprised a removable external tube that can be joined to an internal tube via a T-connector, which was placed close to the naris. Events related to safety (e.g. nasal pressure sores, number of unplanned extubation, displacement and spontaneous migration of the NG tube, other unpredictable injuries) and effectiveness (e.g. liquid food spills, tube obstruction, perfusion rate, other adverse circumstances) were assessed daily.

RESULTS All patients received feeding without complication using the two-piece NG tube and none experienced premature removal of the tube. No serious NG tube complications or malfunctions were observed.

CONCLUSION The results of this study indicate that the two-piece NG feeding tube is a feasible option for patients with dysphagia. Future improvements to the connector may help enhance its performance. A rigorous randomised controlled trial to examine the effects of the two-piece NG tube on patients' quality of life and quality of medical care is being planned.

Keywords: dysphagia, feeding tube, nasogastric, quality of life, rehabilitation

\section{INTRODUCTION}

Patients with severe dysphagia commonly require a nasogastric (NG) tube for feeding and administration of medication. ${ }^{(1)}$ According to a study by Wade, more than $50 \%$ of stroke survivors exhibit lasting complications, including physical swallowing disorders, that necessitate the long-term use of NG tubes. ${ }^{(2)}$ However, previous studies have reported that post-stroke depression is significantly associated with the placement of feeding tubes, as they are perceived to be unsightly and interfere with the activities of daily living. (3) Therefore, when caring for patients who require placement of NG tubes, attention should be paid to the prevention of depression in order to optimise prognosis. ${ }^{(3)}$

Carrión et al reported that the displacement of feeding tubes was associated with poor-quality medical care. ${ }^{(4)}$ According to Neumann et al, repeated placement of feeding tubes is an unnecessary medical expense, a burden on the patient's time and strength, as well as a cause of pain, discomfort, and possibly, harm. ${ }^{(5)}$ Long, exposed feeding tubes can disrupt a patient's daily activities, interfere with treatment, and may even lead to unintentional removal. Burns et al suggested that when the feeding tube was not fixed securely with tape, tube displacement may result. ${ }^{(6)}$ For these reasons, the American College of Critical Care Medicine Task Force has recommended that it may be necessary to use physical restraint to prevent extubation in patients who experience repeated inadvertent tube extubation. ${ }^{(7)}$
Currently, NG tubes differ only in terms of diameter and materials, but not with respect to design or function. We propose that a novel two-piece NG tube might not only improve the quality of life of dysphagic patients but also the quality of medical care. A pilot study was conducted to evaluate the feasibility of the two-piece NG tube for patients with dysphagia, taking into account the safety and effectiveness of its use.

\section{METHODS}

In this observational study, ten patients (seven inpatients and three outpatients) with severe dysphagia requiring an NG tube were recruited between November 2011 and May 2012 from the Department of Physical Medicine and Rehabilitation, Changhua Christian Hospital, Chunghua, Taiwan. Adult patients aged $\geq 18$ years diagnosed with dysphagia who had an NG tube placed were eligible for participation. Patients were excluded from the study if they were unconscious, unable to follow oral instructions or unable to sign a consent form. Patients with unstable clinical status (i.e. systolic blood pressure $<90 \mathrm{~mm} \mathrm{Hg}$, urine output $<30 \mathrm{~mL} /$ hour, heart rate $<45$ or $>125$ beats per minute, or respiratory rate $<10$ or $>30$ breaths per minute) and patients using a traditional nasogastric (T-NG) tube with fixed lengths of $<50 \mathrm{~cm}$ or $>60 \mathrm{~cm}$ were also excluded. The study was approved by the institutional review board, and written informed consent was obtained from all patients prior to participation.

\footnotetext{
${ }^{1}$ Department of Physical Medicine and Rehabilitation, ${ }^{2}$ Department of Neurology, Changhua Christian Hospital, Changhua, ${ }^{3}$ School of Medicine, Chung Shan Medical University, Taichung, Taiwan

Correspondence: Dr Tsung-Ju Wu, Consultant, Department of Physical Medicine and Rehabilitation, Changhua Christian Hospital, 135 Nanhsiao Street, Changhua 500, Taiwan, Republic of China. liusenyong111@yeah.net
} 


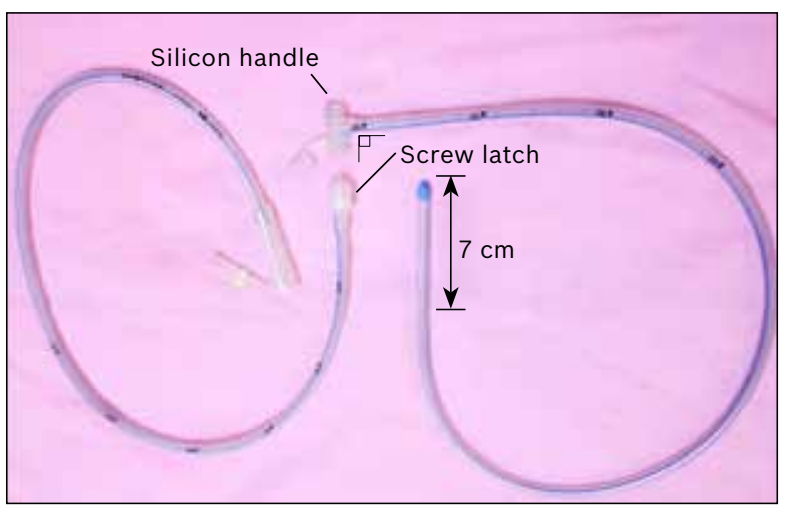

Fig. 1 Photograph shows the novel two-piece nasogastric tube, which comprises a removable external tube (left) and an internal tube (right) that is joined to a T-connector (arrow). The length of a typical adult NG tube from the front end to the last hole is about $7 \mathrm{~cm}$.

Participating patients were fitted with a two-piece tube and evaluated daily for one week on the safety and effectiveness of the NG tube. Inpatients were assessed in person, while outpatients were assessed via telephone calls by a physician who was not part of the study. Events related to safety (e.g. nasal pressure sores, number of unplanned extubation, displacement and spontaneous migration of the NG tube, and other unpredictable injuries) and effectiveness (e.g liquid food spills, tube obstruction, perfusion rate and other adverse circumstances) were assessed. The two-piece NG tube was replaced with the T-NG tube at the end of the study by the original attending physician. For patients whose swallowing function was restored prior to the completion of the study, it was agreed that the twopiece NG tube would be removed earlier.

Ten sets of the two-piece NG tube $(100 \%$ medical-grade silicone, 16Fr; Fortune Medical Instrument Corp Taipei, Taiwan) were prepared. The apparatus (Fig. 1) comprises a removable external tube that can be attached to an internal tube via a T-connector, which is placed close to the naris. One end of the $\mathrm{T}$-connector connects out at $90^{\circ}$, while the other end is joined to an extended silicon handle. There is a screw latch at the site where the external tube is secured to the T-connector, thus ensuring a tight connection between the two tubes to prevent leakage of air or liquid. The extended silicon handle of the T-connector serves to secure the adhesive tape, fasten the external connector to the internal tube and prevent the internal tube from entering the nostril (Fig. 2).

The procedure for inserting the two-piece NG tube was the same as that for a T-NG tube - a bubble test was performed to confirm that the tube was in the stomach. ${ }^{(8)}$ However, the method of fixation was different in that the adhesive tape was secured on the silicon handle of the T-connector for the two-piece NG tube. The length of the two-piece NG tube to be inserted was determined prior to placement, based on the length of the tubing used for a T-NG tube $-55 \mathrm{~cm}$ or $60 \mathrm{~cm}$. The length of the internal tubing was allowed to be up to $5 \mathrm{~cm}$ longer than desired, but not shorter, to prevent backflow of food into the trachea. For this reason, patients who required internal tubing $<50 \mathrm{~cm}$ or
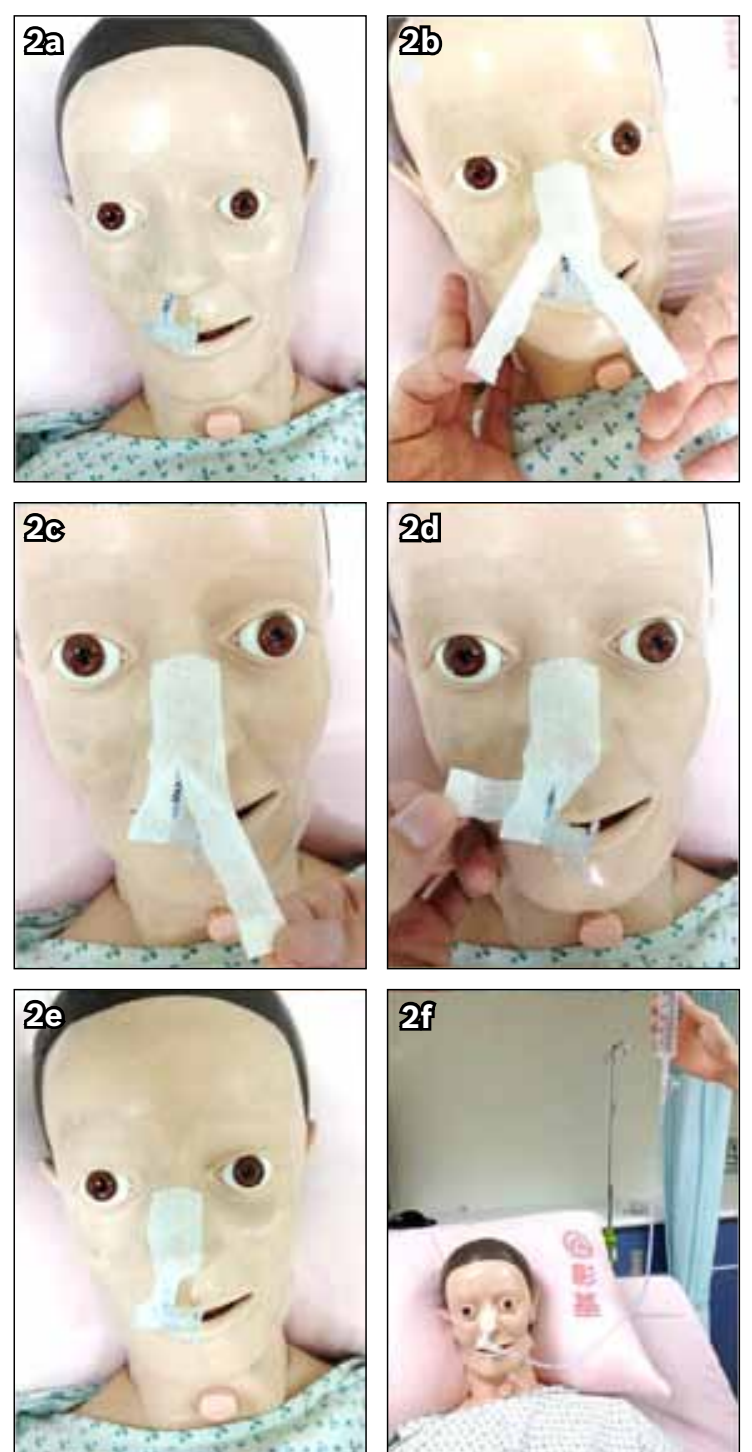

Fig. 2 Photographs show the procedure for fixation of the nove two-piece nasogastric tube. (a) Place the T-connector close to the naris. (b) Prepare a piece of Y-shaped adhesive tape (with two tails) and secure the uncut end of the tape to the nose. (c) Wrap one end of the Y-shaped tape around the silicon handle. (d) Wrap the other end of the tape around the tube using a $90^{\circ}$ rotation. (e) Stick the two ends of the Y-shaped tape securely together. (f) Connect the external tube to the T-connector for feeding.

$>60 \mathrm{~cm}$ were excluded from the study. The connector was T-shaped, with the widest part of the connector measuring $2.35 \mathrm{~cm}$, which helped to prevent spontaneous migration into the nasal cavity under normal conditions. After placement of the two-piece NG tube, caregivers were given instructions on proper care. The procedure for connecting the external and internal pieces of the two-piece NG tube was similar to that for connecting a T-NG tube to the feeding syringe. The external tube was removed during non-feeding periods. In patients who required stomach decompression due to disease, the drainage bag was retained by connecting to the external tube, similar to the case of a T-NG tube.

\section{RESULTS}

A total of 15 patients were screened between November 2011 and May 2012. Of these, five patients were excluded; 
Table I. Patient demographics.

\begin{tabular}{llllll}
\hline Patient no. & Age $(\mathbf{y r s}) /$ gender & Disease & Inpatient & T-NG tube fixation (cm) & Two-piece NG tube fixation (cm) \\
\hline 1 & $53 /$ male & CVA & Yes & 58 & 60 \\
2 & $70 /$ male & Pharyngeal cancer & No & 60 & 60 \\
3 & CVA & No & Yes & 53 & 55 \\
4 & CVA & Yes & 58 & 55 \\
5 & $77 /$ female & CVA & Yes & 57 & 60 \\
6 & $66 /$ male & CVA & Yes & 54 & 50 \\
7 & $88 /$ female & CVA & No & 54 & 55 \\
8 & $88 /$ male & CVA & Yes & 53 & 55 \\
10 & $94 /$ female & $75 /$ female & Parkinson's disease & Yes & 54 \\
\hline
\end{tabular}

CVA: cerebrovascular accident; NG: nasogastric; T-NG: traditional nasogastric

four failed to meet the inclusion criteria and one opted not to participate in the study. Among the remaining ten patients enrolled in the study, six were women and four were men. The average age of the cohort was 76.9 (range 53-94) years. The causes of dysphagia in our cohort were stroke $(n=8)$, pharyngeal cancer $(n=1)$ and Parkinson's disease $(n=1)$. The patient demographics are presented in Table I.

No premature removal of the two-piece NG tube was encountered in our patients, and all ten patients completed the one-week study. No safety events were observed, including nasal pressure sores, unplanned extubation or migration of the tubing into the body. In one patient, a single 5-cm tubing dislodgment occurred during sneezing, the cause of which was ascertained to be poorly secured tape. The tubing was subsequently repositioned and fixed with tape by a family member. Examination by the researchers determined that no replacement tubing was required in this patient.

Four minor effectiveness events were reported. One patient experienced a single episode of liquid feed leakage because the connector was not tightened sufficiently, two patients reported a single instance of temporary obstruction at the connector following the administration of drugs that were not ground finely enough, and one patient reported a single episode of slow flow rate due to the administration of fluid of a higher viscosity. No other adverse situations were reported and no NG tube replacement was required in any of the patients.

The two-piece NG tube was well received by the participants, who were able to receive feeds without complications. At the end of the study, all the caretakers reported that the twopiece NG tube was more convenient than the T-NG tube, mainly due to the ease of removal of the external tube, which greatly facilitated their caretaking activities. The patient with oral cancer also reported improved emotional response and quality of life with the two-piece NG tube, and he was more willing to participate in social activities than when he was using the T-NG tube.

\section{DISCUSSION}

In this study, only adult patients who required long-term use of an NG tube and for whom the confirmed length of the original
T-NG tube was appropriate, were selected. The two-piece NG tube was inserted according to the instructions generally followed for T-NG tube placement, although a different fixation protocol was used. We found no safety issues with the two-piece NG tube in patients with dysphagia, and the results of our study support the feasibility of the device in this patient population.

The length of the internal tube and position of the NG tube are recognised as major safety concerns. The length of the NG tube required is generally determined in relation to the surface location and height, ${ }^{(8-1)}$ such as the distance from the nose to the earlobe and to the xiphoid process. Correct assessment of the feeding tube length is important because the risk of aspiration is increased if the proximal end of the NG tube extends only to the oesophagus or gastroesophageal junction. ${ }^{(12)}$ For this reason, determining the proper length and position of the inserted NG tube is critical. ${ }^{(4)} \mathrm{A}$ previous study suggested that the best drainage results were obtained when the placement length of an NG tube was $55-65 \mathrm{~cm} .{ }^{(10)}$ The length of a typical adult NG tube from the front end to the last hole is about $7 \mathrm{~cm}$. Appropriately, placement of the front end of the NG tube into the stomach should be within the desired distance. In our study, the length of the internal tube was selected within $5 \mathrm{~cm}$ of the estimated intubation length.

Clinically, patients who require NG feeding for more than six weeks are recommended to undergo gastrostomy or jejunostomy. ${ }^{(13)}$ A previous study suggested that among patients with dysphagia subsequent to stroke, those who received endoscopic gastrostomy had a lower mortality rate $(13 \%$ vs. $57 \%$ ) and more weight gain ( $77 \%$ vs. $12 \%$ ) when compared to similar patients who remained on NG tube feeding. ${ }^{(14)}$ According to Bannerman et al, patients treated with endoscopic gastrostomy reported a better quality of life, possibly because gastrostomy is more convenient and has less affect on the activities of daily living than an NG tube. ${ }^{(15)}$ An exposed T-NG tube may affect daily activities such as bathing, turning and sleeping. Unlike the T-NG tube, the two-piece NG tube is similar to gastrostomy in that it has no exposed tubing. Most patients and families in our study reported significant improvement in the patient's daily activities with the two-piece NG tube.

Studies have suggested that jejunostomy and gastrostomy are notably more advantageous than NG feeding for patients who 
require long-term artificial nutrition. ${ }^{(14,15)}$ However, a study by Lin et al reported that $93.4 \%$ of patients who required long-term artificial nutrition at home received an NG tube rather than jejunostomy or gastrostomy, mainly because of the patients' inability to undergo the procedures due to advanced age. ${ }^{(16)}$ In an earlier study, Huang et al found that long-term use of an NG tube could cause physical discomfort and lower self-esteem in patients, possibly contributing to post-stroke depression and hindering both rehabilitation success as well as functional prognosis. ${ }^{(3)}$ It is likely that the impact on social activities and mood may be related to the exposed NG tube, especially in fully conscious patients with normal cognition. Such associations were reinforced by the dramatic improvements in emotional wellbeing reported by the patient with oral cancer in our study.

NG tube dislodgment may cause serious complications, as aspiration is likely to occur once the entire front end is in either the oesophagus or gastroesophageal junction. ${ }^{(12)}$ Different methods of tube fixation can also affect the risk of NG tube dislodgment. ${ }^{(7)}$ The most common fixation method involves securing a piece of adhesive tape to the bridge of the nose and wrapping the tape around the NG tube near the naris. Using this method, the NG tube can become dislodged because of nasal secretions, resulting in displacement of the tube, which can be easily overlooked by caregivers. The fixation method used for the two-piece NG tube is more secure since the tape is wrapped around the T-connector. In addition, any dislodgement of the tube is easily observable due to the obvious change in the position of the T-connector. Although one patient in our study experienced displacement of an improperly secured two-piece NG tube due to sneezing, this dislodgment was quickly discovered by a family member and improper feeding was avoided.

Unplanned extubation, which often occurs in restless or uncooperative patients, can lead to repeated replacement of the NG tube, a process which is not only uncomfortable but also potentially risky. ${ }^{(17)}$ According to previous studies, in patients who require long-term use of an NG tube, the most common reason for extubation at home is self-removal $(48.8 \%) .{ }^{(18)}$ Prevention of inadvertent extubation is one of the main reasons for protective restraint in such patients. ${ }^{(6)}$ However, patients and their families may not be amenable to protective restraint, as seen in one hospitalised participant in our group who used to repeatedly and inadvertently pull his NG tube out during sleep, prior to being enrolled into this study. When his T-NG tube was replaced with the two-piece NG tube for this study, no instance of extubation occurred, and both the patient and his family expressed a high degree of satisfaction with the device.

Another advantage of this novel two-piece NG tube is that the external tube can be removed for cleaning and disinfection purposes. However, one disadvantage is the possibility of an unsecured connection causing leakage of feed. In addition, one design flaw of the two-piece NG tube is the $90^{\circ}$ angle of the connector, which has a slightly reduced diameter that can impede the flow of drugs or feed - two patients in our study experienced temporary obstruction at the connector because the drugs administered were not ground finely enough, while one patient reported a slow flow rate when relatively thick fluids were administered. Improvements to the design of the connector should solve these problems.

This study was limited by its small sample size and short study period. A randomised controlled trial with a greater number of patients and a longer study period would help to further examine and establish the safety and effectiveness of the two-piece NG tube.

We conclude that the novel two-piece NG tube is feasible for use in adult patients with dysphagia. Future efforts will focus on refining the design of the tube connector and conducting a rigorous randomised controlled trial that includes more participants over a longer time period.

\section{ACKNOWLEDGEMENT}

The study was supported by an independent grant from the Changhua Christian Hospital, Changhua, Taiwan.

\section{REFERENCES}

1. Stroud $M$, Duncan $H$, Nightingale J. Guidelines for enteral feeding in adult hospital patients. Gut 2003; 52(Suppl 7):vii1-vii12.

2. Wade DT. Measuring arm impairment and disability after stroke. Int Disabil Stud 1989; 11:89-92.

3. Huang NC, Wei TS, Liu SY. The influence of nasogastric tube to post-stroke depressive tendency and functional outcome. Taiwan J Phys Med Rehabil 2006; 34:19-26.

4. Carrión MI, Ayuso D, Marcos M, et al. Accidental removal of endotracheal and nasogastric tubes and intravascular catheters. Crit Care Med 2000; 28:63-6.

5. Neumann MJ, Meyer CT, Dutton JL, Smith R. Hold that x-ray: aspirate $\mathrm{pH}$ and auscultation prove enteral tube placement. J Clin Gastroenterol 1995; 20:293-5.

6. Burns SM, Martin M, Robbins V, et al. Comparison of nasogastric tube securing methods and tube types in medical intensive care patients. Am J Crit Care 1995; 4:198-203.

7. Maccioli GA, Dorman T, Brown BR, et al. Clinical practice guidelines for the maintenance of patient physical safety in the intensive care unit: use of restraining therapies--American College of Critical Care Medicine Task Force 2001-2002. Crit Care Med 2003; 31:2665-76.

8. Dornan T, O’Neill P. Core Clinical Skills for OSCEs in Medicine. 2nd ed. Edinburgh: Churchill Livingstone, 2006:241-2.

9. Price B. Making sense of nasogastric intubation. Nurs Times 1989; 85:50-2.

10. Zhao YH. Methods of increasing the successful rate of one-time nasogastric tube placement and improving the effectiveness of drainage. Nurs Pract Res 2009; 16:1-2.

11. Beckstrand J, Cirgin Ellett ML, McDaniel A. Predicting internal distance to the stomach for positioning nasogastric and orogastric feeding tubes in children. J Adv Nurs 2007; 59:274-89.

12. Metheny NA. Titler MG. Assessing placement of feeding tubes. Am J Nurs 2001; 101:36-45.

13. Yamada T. Textbook of Gastroenterology. 4th ed. Philadelphia: Lippincott Williams \& Wilkins 2003: 1062-3.

14. Norton B, Homer-Ward M, Donnelly MT, Long RG, Holmes GK. A randomised prospective comparison of percutaneous endoscopic gastrostomy and nasogastric tube feeding after acute dysphagic stroke. BMJ 1996; 312:13-6.

15. Bannerman E, Pendlebury J, Phillips F, Ghosh S. A cross-sectional and longitudinal study of health-related quality of life after percutaneous gastrostomy. Eur J Gastroenterol Hepatol 2000; 12:1101-9.

16. Lin LC, Li MH, Watson R. A survey of the reasons patients do not chose percutaneous endoscopic gastrostomy/jejunostomy (PEG/PEJ) as a route for long-term feeding. J Clin Nurs 2011; 20:802-10.

17. Metheny NA, Clouse RE, Chang YH, et al. Tracheobronchial aspiration of gastric contents in critically ill tube-fed patients: frequency, outcomes, and risk factors. Crit Care Med 2006; 34:1007-15.

18. Ho MM, Hor YS, Li SC, et al. The exploration of home care patients' unplanned extubation and the primary caregivers' knowledge, and learning needs related to tubing care. J Long-term Care 2008; 12:72-90. 